\title{
Tailoring the switch from IRES-dependent to 5 '-end-dependent translation with the RNase P ribozyme
}

\author{
NOEMI FERNÁNDEZ and ENCARNACIÓN MARTÍNEZ-SALAS \\ Centro de Biología Molecular Severo Ochoa, Consejo Superior de Investigaciones Científicas-Universidad Autónoma de Madrid, Cantoblanco \\ 28049 Madrid, Spain
}

\begin{abstract}
Translation initiation driven by internal ribosome entry site (IRES) elements is dependent on the structural organization of the IRES region. We have previously shown that a structural motif within the foot-and-mouth-disease virus IRES is recognized in vitro as substrate for the Synechocystis sp. RNase P ribozyme. Here we show that this structure-dependent endonuclease recognizes the IRES element in cultured cells, leading to inhibition of translation. Inhibition of IRES activity was dependent on the expression of the active ribozyme RNA subunit. Moreover, expression of the antisense sequence of the ribozyme did not inhibit IRES activity, demonstrating that stable RNA structures located upstream of the IRES element do not interfere with internal initiation. RNAs carrying defective IRES mutants that were substrates of the ribozyme in vivo revealed an increased translation of the reporter in response to the expression of the active ribozyme. In support of RNA cleavage, subsequent analysis of the translation initiation manner indicated a switch from IRES-dependent to 5 ' -end-dependent translation of RNase P target RNAs. We conclude that the IRES element is inactivated by expression in cis of RNase P in the cytoplasm of cultured cells, providing a promising antiviral tool to combat picornavirus infections. Furthermore, our results reinforce the essential role of the structural motif that serves as RNase $P$ recognition motif for IRES activity.
\end{abstract}

Keywords: translation initiation; IRES; RNase P ribozyme; RNA virus; picornavirus

\section{INTRODUCTION}

Translation initiation of picornavirus RNAs occurs by a cap-independent process, mediated by internal ribosome entry site (IRES) elements (Martinez-Salas et al. 2008). The IRES of the foot-and-mouth disease virus (FMDV), which is the prototypic member of the aphthovirus genus in the Picornaviridae family, has been studied by combining functional analysis with RNA probing and RNA-protein interactions (Fernandez-Miragall et al. 2009). The FMDV IRES is organized in three distinct regions. The $5^{\prime}$ and $3^{\prime}$ regions (encompassing domains 2 and $4-5$, or $\mathrm{H}$ and $\mathrm{JK}-\mathrm{L}$, respectively) are involved in RNA-protein interactions including translation initiation factors (eIFs) (Lopez de Quinto and Martinez-Salas 2000; Pilipenko et al. 2000; Lopez de Quinto et al. 2001; Andreev et al. 2007) and auxiliary proteins (Song et al. 2005; Monie et al. 2007; Pacheco

Reprint requests to: Encarnación Martínez-Salas, Centro de Biología Molecular Severo Ochoa, Consejo Superior de Investigaciones CientíficasUniversidad Autónoma de Madrid, Cantoblanco 28049 Madrid, Spain; e-mail: emartinez@cbm.uam.es; fax: 34911964420.

Article published online ahead of print. Article and publication date are at http://www.rnajournal.org/cgi/doi/10.1261/rna.1973710. et al. 2009). The central region is composed of a self-folding domain (termed 3 or I) that includes a conserved GNRA motif (where $\mathrm{G}$ stands for guanine; $\mathrm{N}$, any nucleotide; $\mathrm{R}$, purine; and A, adenine) and mediates intramolecular RNARNA interactions (Ramos and Martinez-Salas 1999; FernandezMiragall et al. 2006).

We have shown previously that the FMDV IRES contains a structural motif that is recognized in vitro by the RNA subunit of Synechocistis sp. RNase P. One main single cleavage site was observed with overlapping RNA substrates, either the full-length IRES or short transcripts encompassing the apical region of domain 3 (Serrano et al. 2007). No cleavages were found in the downstream 3' regions of the IRES. RNA hydrolysis was dependent on high $\mathrm{Mg}^{2+}$ concentration and yielded cleavage products with $5^{\prime}-\mathrm{P}$ and 3'-OH ends, consistent with a specific RNase P cleavage (Robertson et al. 1972; Vioque 1992). Alignment of the hydrolysis products derived from overlapping substrates as well as primer extension analysis of the reaction products mapped the cleavage site toward $\mathrm{U}_{169}-\mathrm{C}_{172}$ of the FMDV IRES (Serrano et al. 2007). These residues are located in a bulge within the GNRA stem-loop. According to RNA probing analysis, the GNRA motif adopts a tetraloop 
conformation and determines the local RNA structure of domain 3, affecting also the RAAA stem-loop (FernandezMiragall and Martinez-Salas 2003; Fernandez-Miragall et al. 2006). Our studies demonstrated that defective IRES elements with a modified RNA structure in the apical region of the central domain responded in a different manner to ribozyme cleavage (Serrano et al. 2007).

RNase $\mathrm{P}$ is an endonuclease involved in the processing of tRNA precursor in all organisms (Evans et al. 2006; Ellis and Brown 2009; Kirsebom and Trobro 2009). However, in the last few years, a large number of non-tRNA RNase $P$ substrates have been described using a variety of experimental settings (Alifano et al. 1994; Nadal et al. 2002; Li and Altman 2003; Lyons and Robertson 2003; Ko and Altman 2007; Yang and Altman 2007; Coughlin et al. 2008). The RNase P ribozyme from Synechocystis sp., which does not require the CCA sequence at the $3^{\prime}$ end of the tRNA precursor for its hydrolysis (Pascual and Vioque 1999), also recognizes the hepatitis $\mathrm{C}$ virus (HCV) IRES in vitro (Sabariegos et al. 2004). However, no cleavage of the viral RNA in infected or transfected cells has been observed by the endogenous nuclear RNase P (Piron et al. 2005), consistent with the fact that the viral cycle occurs in the cytoplasm of infected cells. The biological significance of the RNase $\mathrm{P}$ recognition motif within IRES elements is still unknown.

Here we have used constructs that express the RNase P ribozyme from Synechocystis sp. as a tool to investigate its capacity to interfere with IRES activity in tissue culture cells. We have found that FMDV IRES activity was strongly inhibited by the expression in cis of the active ribozyme concomitant with RNA cleavage. Expression of a mutant RNase $\mathrm{P}$ or the antisense sequence of the $r p p B$ gene did not induce RNA cleavage nor inhibit translation. Interestingly, and in support of the specific cleavage of the IRES element by the cyanobacterial RNase P, the study of transcripts carrying defective IRES elements that were substrates of this ribozyme revealed a switch in the translation initiation mechanism from IRES dependent to 5 '-end dependent.

\section{RESULTS}

\section{The cyanobacterial RNase P RNA subunit induces cleavage of IRES elements in tissue-cultured cells}

We have previously shown that, in vitro, the Synechocystis PCC6803 RNase P ribozyme recognizes as substrate a structural motif located within domain 3 of the FMDV IRES yielding two main cleavage products (Serrano et al. 2007). RNA hydrolysis, however, was detected only in about $10 \%$ of the IRES substrate. To test the capacity of this ribozyme to recognize the IRES element in the cellular cytoplasm we generated a construct, designated Rz-IRES, which encodes in a single transcriptional unit the $r n p B$ gene (RNase $\mathrm{P}$ ribozyme) from Synechocystis sp. PCC6803 (Vioque 1992), upstream of the FMDV IRES (Fig. 1A). As a control of a similarly organized transcriptional unit but lacking ribozyme activity, we generated two additional constructs, RzP4-IRES and asRz-IRES. The first one, RzP4-IRES, carries a double nucleotide substitution $\left(\mathrm{GG}_{414-415}\right.$ to $\left.\mathrm{CC}\right)$ that impairs base pairing within the conserved helix $\mathrm{P} 4$ of the RNase P RNA structure (Pace and Brown 1995; Ellis and Brown 2009), negatively affecting ribozyme activity. The second one, asRz-IRES, contains the $\operatorname{rnp} B$ gene inserted

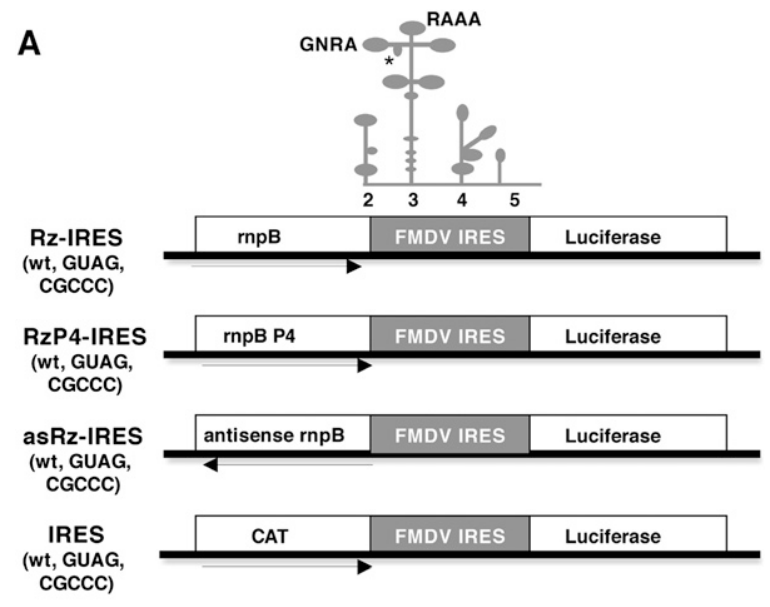

B
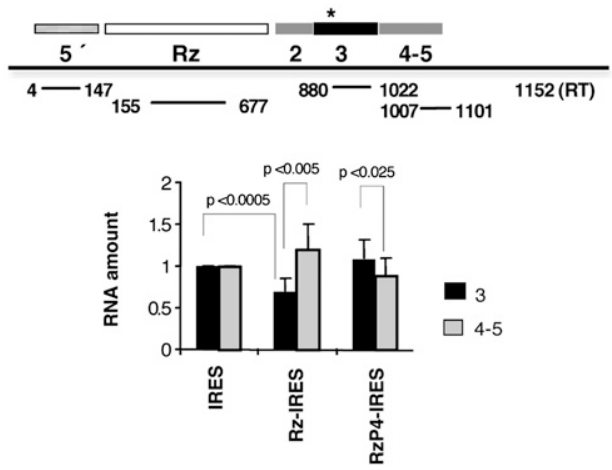

FIGURE 1. (A) Diagram of ribozyme-IRES constructs. Three versions of the RNase P ribozyme (PCC6803 rnpB wild type, the GG to CC mutation in helix P4, and the antisense orientation of the $r n p B$ gene) were combined with three different sequences of the IRES element (the wild type, the GUAG mutation in the GNRA motif, and the CGCCC mutation in the RAAA motif). A schematic representation of the FMDV IRES structure with indication of the different domains, the location of the GNRA and RAAA motifs, and the RNase $P$ cleavage site (depicted by an asterisk) mapped in vitro is represented at the top. CAT and luciferase indicate the reporter genes. $(B)$ Determination of reporter RNA amount. Real-time RT-PCR analysis of reporter RNAs in cells transfected with the different versions of the ribozyme constructs. The amount of RNA corresponding to domain 3 (nucleotides 880-1022, relative to the +1 residue of the reporter RNA) or domains 4-5 (nucleotides 1007-1101) of the FMDV IRES was determined in duplicate assays of three independent experiments by RT-qPCR and made relative to the amounts of the $5^{\prime}$ sequence (nucleotides 4-147) present in the same samples. Error bars indicate the standard deviation. The Student's $t$-test was used for statistical calculation, and $P$ values are indicated. 
in the negative orientation upstream of the IRES element (Fig. 1A).

To assess the ability of the $\mathrm{rnpB}$ ribozyme to recognize as substrate the FMDV IRES in vivo, BHK-21 cells were transfected with Rz-IRES and RzP4-IRES in parallel with the pBIC-IRES construct (Fig. 1A), using the T7 RNA polymerase expression system (Martinez-Salas et al. 1993). The amount of uncleaved RNA present in the cell cytoplasm $4 \mathrm{~h}$ post-transfection (hpt) was determined by RT-qPCR using total RNA purified from cells transfected with each construct. The amplification efficiency of a sequence very close to the $5^{\prime}$ end of the transcript (Fig. 1B, nucleotides 4-147) was used in all cases to normalize for the differences in the amounts of RNA transcribed from the transfected construct. The amplification control performed in the absence of RT (Supplemental Fig. S1) confirmed the absence of DNA contaminating the RNA sample. The relative abundance of RNA corresponding to two different regions of the FMDV IRES, domain 3 (Fig. 1B, nucleotides 880-1022, relative to +1 position of the transcriptional unit), which contains the RNase $\mathrm{P}$ recognition motif (Serrano et al. 2007), and domains 4-5 (Fig. 1B, nucleotides 1007-1101) was determined by RT-qPCR performing duplicate analysis of at least two independent samples. As shown in Figure 1B, a decrease of about $40 \%$ in the amount of RNA corresponding to domain 3 was observed in the samples derived from cells transfected with construct Rz-IRES relative to the cells transfected with the pBIC vector that encodes chloramphenicol acetyl transferase (CAT) instead of the ribozyme in the first cistron (Fig. 1A). By contrast, no decrease was observed in the RNA region corresponding to domains 4-5, suggesting that extensive RNA degradation did not occur at this time post-transfection. Although there was some variability in the amount of RNA detected by RTqPCR in independent transfection assays, the $P$ values obtained in the Student $t$-test supported its statistical significance. No decrease in the relative abundance of the same IRES domains was observed in samples derived from cells transfected with construct RzP4-IRES (Fig. 1B), demonstrating absence of ribozyme activity in this RNA. Thus, we conclude that expression of the rnpB ribozyme in construct Rz-IRES was responsible for partial cleavage of the IRES RNA.

\section{IRES activity is inhibited by expression of the Synechocystis RNase P ribozyme}

Next, to test the capacity of this ribozyme to interfere with IRES activity, we determined the translation efficiency of the transcriptional unit bearing the Synechocystis rnpB ribozyme upstream of the FMDV IRES that drives translation of the firefly luciferase reporter (Fig. 1A). To this end, equal amounts of plasmids pBIC and Rz-IRES were transfected in BHK-21 cells, and the expression of luciferase, measured at different times post-transfection, was made relative to the total amount of protein in the extract. In comparison with the construct that expresses CAT as the first cistron (cap-dependent) and luciferase as the second cistron (IRES-dependent), transfection of the Rz-IRES construct led to a strong inhibition of IRES activity (Fig. 2A). Inhibition of IRES activity in construct Rz-IRES was detected very early upon transfection, and it was maintained over $24 \mathrm{~h}$. A clear trend to inhibit IRES activity was observed within a range of twofold lower amounts of transfected construct (Supplemental Fig. S2).

Expression of the rnpB RNA could interfere with internal initiation of translation through an event unrelated to the ribozyme activity. Thus, to test this possibility we analyzed

A

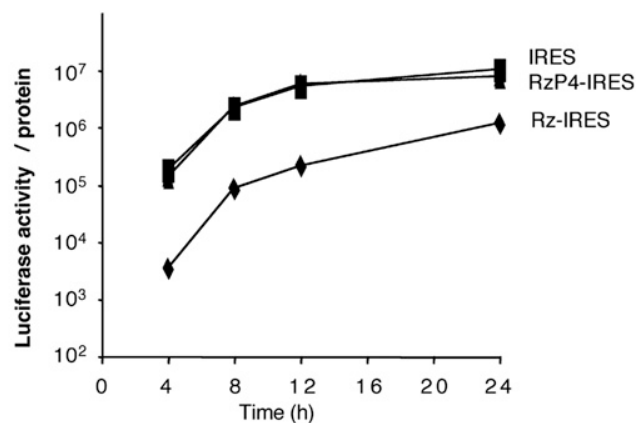

B
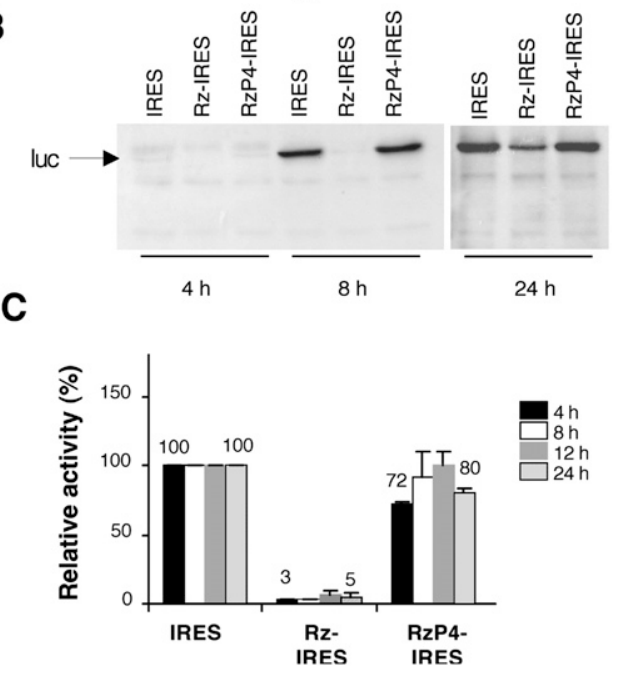

FIGURE 2. Expression of the active rnpB ribozyme inhibits IRES activity. (A) Time course of reporter translation in response to expression of the rnpB ribozyme. The luciferase activity (measured as relative light units, RLU) of extracts prepared from cells transfected with the indicated constructs $\left(0.33 \mu \mathrm{g} / 10^{5}\right.$ cells $)$ was determined over a period of $24 \mathrm{~h}$ and normalized to the concentration of protein in the extract. Values of luciferase activity/protein correspond to the average obtained from triplicate wells and performed in at least three independent assays. (B) Immunodetection of luciferase expression by Western blot. $(C)$ Levels of inhibition of IRES-dependent translation by expression of the RNase P ribozyme. The luciferase activity determined at the indicated time in extracts of cells transfected with the ribozyme constructs was normalized to the value observed in the IRES construct set to $100 \%$. Error bars correspond to the standard deviation. 
IRES activity in cells transfected with the construct RzP4IRES that encodes an RNA differing from the wild type in two bases involved in the formation of helix $\mathrm{P} 4$ required for hydrolysis (Vioque 1992; Pace and Brown 1995). In this construct, transcription from the T7 promoter leads to the synthesis of an RNA of the same length than Rz-IRES but lacking ribozyme activity (Fig. 1B). Remarkably, transfection of RzP4-IRES resulted in negligible inactivation of IRES activity in comparison with the control IRES, irrespective of the time post-transfection used to measure expression of the reporter (Fig. 2A). Similar conclusion was derived from the analysis of reporter expression by Western blot (Fig. 2B).

The decrease in relative activity induced by expression of Rz-IRES in three independent assays reached values around $5 \%$ of the wild type (Fig. 2C). Instead, the activity measured in cells transfected with RzP4-IRES remained fairly constant, with relative values around $80 \%$ of the wild type. Thus, inhibition of IRES activity was specifically due to the expression of the wild-type $r m p B$ gene.

\section{Inhibition of internal initiation is unrelated to the presence of stable RNA structure upstream of the IRES element}

Next, to discard the possibility that insertion of a highly stable RNA structure upstream of the IRES element was inhibitory for internal initiation of translation, we analyzed IRES activity in cells transfected with construct asRz-IRES that encodes the antisense sequence of the $r m p B$ gene upstream of the IRES element. RNA structure prediction of the rnpB antisense sequence (http://mfold.bioinfo.rpi.edu/ cgi-bin/dna-form1.cgi) indicated the presence of extensively paired RNA, with an internal energy similar to that of the rnpB RNA subunit (data not shown). Transfection of asRz-IRES did not alter IRES activity in comparison with the control IRES construct, irrespective of the time posttransfection used to measure expression of the reporter (Fig. 3A,B). As expected, RT-qPCR analysis did not reveal differences in RNA amounts corresponding to domain 3 or domains $4-5$ (Fig. 3C). We conclude that IRES activity is unaffected by stable RNA structures upstream of the IRES element.

\section{RNA cleavage of severely defective IRES mutants leads to translation increase}

The results shown above indicated that IRES activity was efficiently inhibited even though cleavage of Rz-IRES RNA was partial. Therefore, translation of the reporter measured in Rz-IRES transfected cells (Fig. 2A) could arise by two initiation mechanisms, $5^{\prime}$-dependent in cleaved RNAs and IRES-dependent in uncleaved RNAs. To test this possibility and validate the conclusions obtained with the wild-type IRES, we fused the $\operatorname{rpp} B$ gene to the cDNA encompassing two different defective IRES mutants (carrying sequences
A

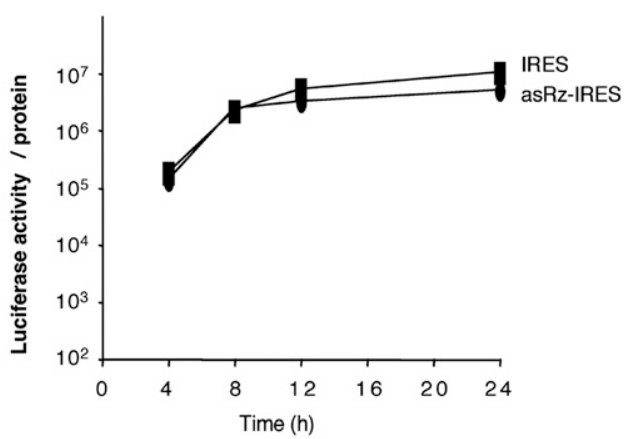

B
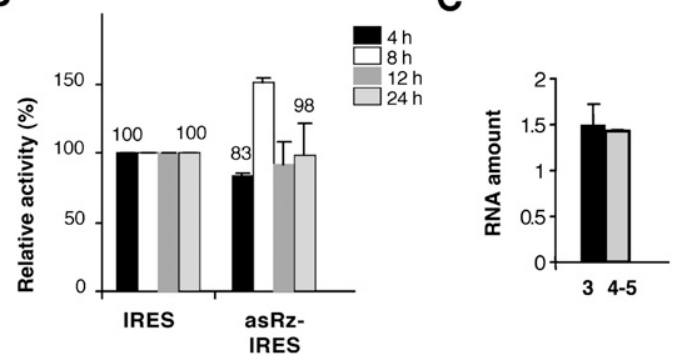

FIGURE 3. Insertion of structured RNA upstream of the IRES region does not affect IRES activity. (A) Time course of reporter translation efficiency in construct expressing the antisense sequence of the rnpB ribozyme upstream of the IRES region. Values correspond to the average of luciferase activity obtained from triplicate wells and performed in three independent assays. $(B)$ Lack of inhibition of IRES-dependent translation by expression of the antisense sequence of rnpB. The luciferase activity determined at the indicated time in extracts of cells transfected with the asRz-IRES construct was normalized to the value observed in the IRES construct set to $100 \%$. Error bars correspond to the standard deviation. (C) Expression of the antisense sequence of the $r m p B$ gene does not induce RNA cleavage. RT-qPCR was used to determine the RNA amount corresponding to domains 3 and $4-5$ of the IRES.

GUAG or CGCCC in the GNRA or RAAA motifs, respectively) (Fig. 1A) that initiate translation at about $1 \%$ of the efficiency of the wild-type IRES (Lopez de Quinto and Martinez-Salas 1997). We have shown previously that these defective IRES elements are targets of the Synechocystis RNase P ribozyme in vitro (Serrano et al. 2007). If our working hypothesis proves to be correct, cleavage of defective IRES is expected to increase the efficiency of protein synthesis owing to a change from defective IRES-dependent to a $5^{\prime}$-end-dependent mechanism, in marked contrast to the detrimental effect observed with wild-type IRES. On the contrary, if translation inhibition is unrelated to RNA hydrolysis the activity of the defective IRES RNAs should remain unaffected.

First, RT-qPCR analysis was conducted to verify if the RNA expressed from Rz-GUAG construct was recognized as substrate of the ribozyme in transfected cells. About 50\% decrease in the amount of RNA corresponding to domain 3 , the structural domain recognized by this ribozyme, was detected in Rz-GUAG (Fig. 4A). Instead, no significant changes in the amount of domain 3 RNA were observed in 
A

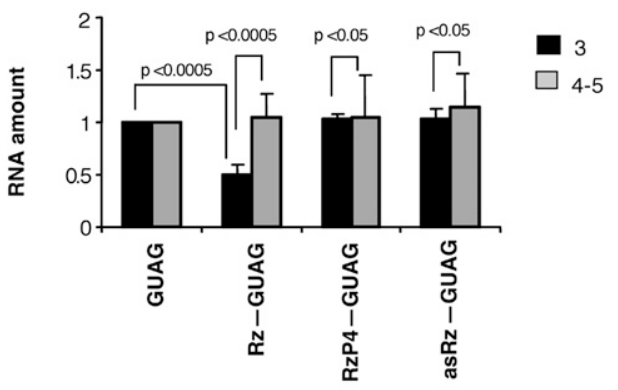

B

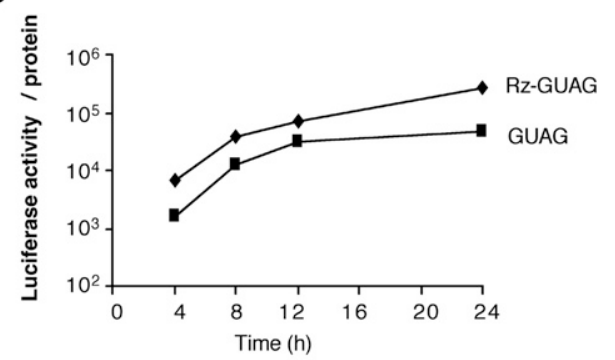

C

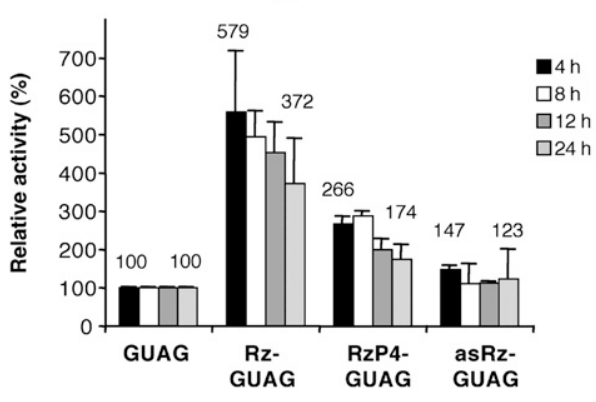

FIGURE 4. RNase P recognition of the defective GUAG IRES leads to increased expression of the reporter. (A) Ribozyme-mediated cleavage of the reporter RNA. Real-time RT-qPCR analysis of reporter RNAs in cells transfected with the different versions of the ribozyme fused to the GUAG IRES. Data are expressed as in Fig. 1B. (B) Time course of reporter translation in response to expression of the rnpB ribozyme, fused to the IRES element carrying the single GUAG mutation in the GNRA motif. Data are represented as in Fig. 2A. (C) Changes in translation levels induced by expression of the RNase $\mathrm{P}$ ribozyme. Data are represented as in Fig. 2C.

samples derived from cells transfected with RzP4-GUAG or asRz-GUAG constructs, again demonstrating the lack of ribozyme activity of these RNAs. Similarly, and consistent with the results observed in the wild-type IRES, the amounts of RNA corresponding to domains $4-5$ of the IRES remained fairly constant in all cases.

The effect on translation in construct Rz-GUAG is shown in Figure 4B. An increase in luciferase activity was observed in Rz-GUAG relative to the values obtained with the defective GUAG IRES (which rendered about $1 \%$ of the wild-type IRES activity, compare with Fig. 2A). The increase in expression of the reporter in Rz-GUAG transfected cells was maintained over a period of $24 \mathrm{~h}$ (Fig. 4B). While the average of three independent assays made relative to the activity observed in the GUAG construct showed about fivefold increase in reporter translation in Rz-GUAG transfected cells, expression of the reporter in construct RzP4-GUAG, and particularly asRz-GUAG, was barely changed (Fig. 4C).

To reinforce the results obtained with the GUAG IRES mutant, we made use of a different defective mutant termed CGCCC (Fig. 1A). This mutant, which displays an IRES activity about $1 \%$ of the wild type, alters the RNA structure of the RAAA stem-loop within the apical region of domain 3 and is also a target of RNase P (Serrano et al. 2007). In agreement with the results shown for Rz-GUAG RNA, the amount of RNA detected by RT-qPCR was lower for domain 3 in the active ribozyme construct (Rz-CGCCC), and remained constant in the inactive ribozyme constructs, RzP4-CGCCC or asRz-CGCCC (Fig. 5A). As before, no decrease was detected in the amount of domain 4-5 RNA. Furthermore, an increase of four- to fivefold in luciferase activity was obtained with this defective mutant when expressed as Rz-CGCCC, but not as the inactive ribozyme RzP4-CGCCC or asRz-CGCCC RNAs (Fig. 5B,C). Thus, the results obtained with two different mutants of the IRES

A

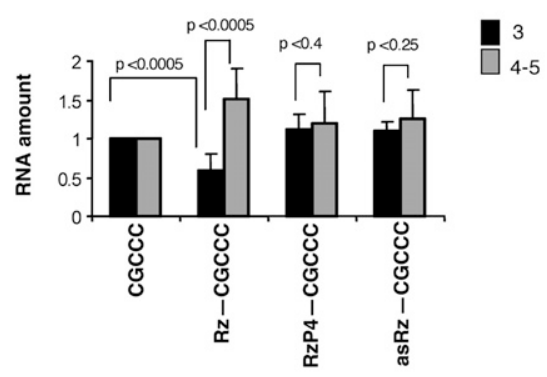

B

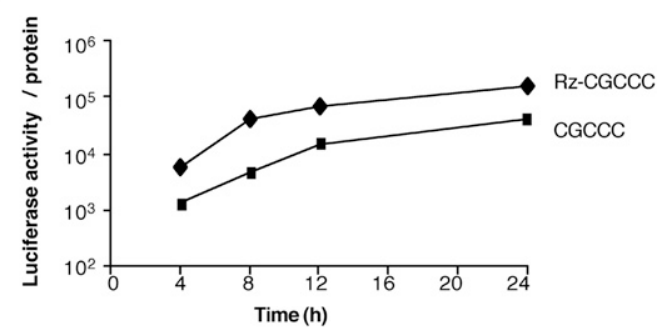

C

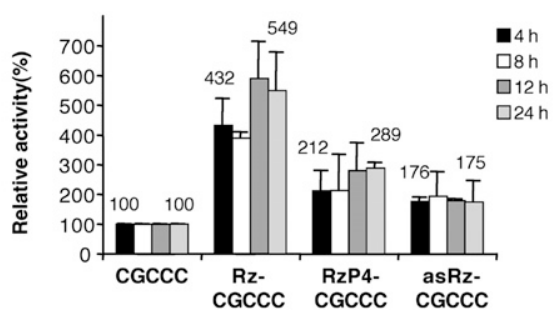

FIGURE 5. RNase $P$ recognition of the defective CGCCC IRES induces an increase in reporter translation. (A) Ribozyme-mediated cleavage of the reporter RNA. Real-time RT-qPCR analysis of reporter RNAs in cells transfected with the different versions of the ribozyme fused to the CGCCC IRES. Data are expressed as in Fig. 1B. (B) Time course of reporter translation in response to expression of the rnpB ribozyme, fused to the IRES carrying a CGCCC sequence in the RAAA motif. Data are represented as in Fig. 2A. (C) Changes in reporter levels induced by expression of the RNase P ribozyme. Data are represented as in Fig. 2C. 
element manifested an increase in translation following hydrolysis of Rz-GUAG and Rz-CGCCC transcriptional units.

\section{RNase $P$ recognition \\ of the IRES induces a switch \\ from 5 '-independent \\ to $5^{\prime}$-dependent translation}

Cleavage of the IRES element is expected to result in a decrease of internal translation accompanied by a change to 5 '-end-dependent initiation, provided that the hydrolyzed RNA is not degraded. Results from RT-qPCR demonstrated that levels of domains 4-5 were similar in samples transfected with constructs IRES, RzP4-IRES or asRzIRES, supporting the idea that there was not extensive degradation of RzIRES RNA under the conditions used in our study. We therefore proceeded to test whether cleavage of the Rz-IRES RNA generated a free $5^{\prime}$-end RNA that could mediate a $5^{\prime}$-end-dependent translation initiation of the luciferase reporter. To this end, we made use of a functional assay. Expression of the Lb protease encoded in the FMDV viral genome in mammalian cells is known to cleave the translation initiation factor eIF4G as well as poly $(\mathrm{A})$-binding protein (PABP) and, thus, to inactivate cap-dependent translation but not IRES-dependent translation (Devaney et al. 1988; Martinez-Salas et al. 1993; Medina et al. 1993, Rodriguez-Pulido et al. 2007). As expected for cap-dependent initiation, cotransfection of a plasmid expressing the Lb protease with the bicistronic IRES plasmid resulted in a decrease of CAT that was detected as early as $4 \mathrm{hpt}$ (Fig. 6A). Instead, luciferase expression was resistant to $\mathrm{Lb}$ expression, in agreement with IRES-dependent initiation. Cotransfection of Rz-IRES and $\mathrm{pLb}$ constructs resulted in an increase of luciferase translation relative to the values obtained in the transfection carried out with Rz-IRES supplemented with the empty vector (Fig. 6B). We interpret this increase as a result of the IRES-dependent translation of uncleaved RNA in the absence of competition with the cap-dependent cellular mRNAs. No decrease in luciferase activity was observed in the cotransfection of RzP4-IRES or asRz-IRES and Lb in comparison with the cotransfection performed with RzIRES supplemented with the empty vector, consistent with IRES-dependent translation of luciferase. In all cases, the response to $\mathrm{Lb}$ expression was similar at two times (4 and $8 \mathrm{~h}$ ) post-transfection (Fig. 6B).

Next, to test whether the increase of luciferase expression driven by the defective GUAG transcriptional unit was due to $5^{\prime}$-end-dependent translation, we made use again of the cotransfection of constructs expressing the defective IRES element with the Lb protease. As shown in Figure 7A, the response to $\mathrm{Lb}$ coexpression was dependent on the RNA sequence upstream of the IRES element. Constructs RzP4GUAG and asRz-GUAG were insensitive to the coexpression of Lb. Instead, a decrease of about twofold in luciferase activity was observed in construct Rz-GUAG relative to the value obtained in its absence. Consistent with the expression of an active Lb protease, the expression of CAT (cap dependent, encoded in the first cistron of the GUAG construct) was significantly inhibited. Similar results were obtained with the IRES mutant CGCCC (data not shown), further validating the results obtained with the defective GUAG IRES. Thus, we conclude that cleavage of the IRES by RNase $\mathrm{P}$ leads to the appearance of a free $5^{\prime}$-end RNA that induces a switch from IRES-dependent to $5^{\prime}$-dependent translation.

In support of this conclusion, primer extension analysis conducted with total RNAs prepared from transfected cells and a specific ${ }^{32} \mathrm{P}$-labeled antisense primer confirmed the presence of new reverse transcriptase (RT) stops in Rz-IRES and Rz-GUAG RNA, which were undetected in the RNAs with the inactive ribozyme (Fig. 7B). Consistent with the RT-qPCR data shown in Figure 4A, RT stops were located at nucleotides A167, C192, and C211 in the Rz-IRES RNA (nucleotide numbers as in Serrano et al. 2007), while a clear RT stop at nucleotide C192 was detected in Rz-GUAG 
B

A
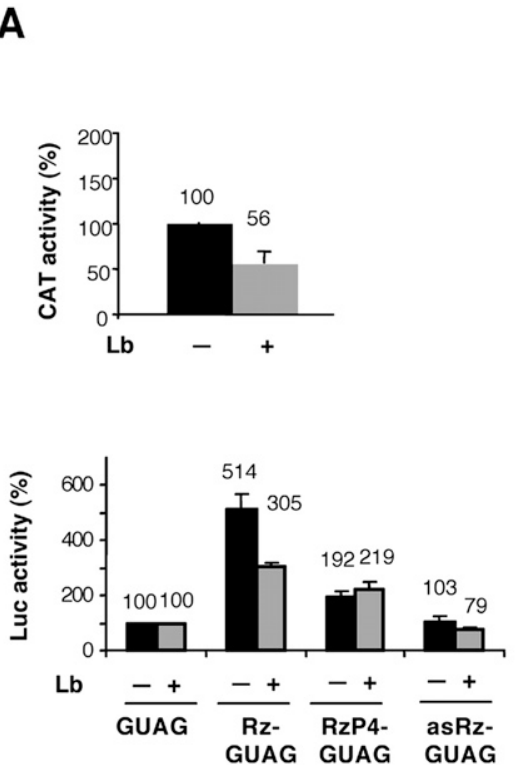

FIGURE 7. (A) Switch from IRES-dependent to $5^{\prime}$-end-dependent translation in defective IRES constructs in response to coexpression of the rnpB ribozyme with the Lb protease. BHK21 cells were transfected with the ribozyme-GUAG IRES constructs in the presence or absence of the Lb-expression plasmid. Lysates collected $4 \mathrm{hpt}$ were used to measure CAT (IRES construct) or luciferase (all constructs). Levels of luciferase activity measured in the ribozymeIRES constructs were made relative to those detected in the extract corresponding to the control GUAG IRES vector (591200 RLU 4 hpt), set to 100\%. (B) Primer extension analysis of the ribozyme cleavage sites. Total RNA isolated from cells transfected with Rz-IRES, RzP4IRES, Rz-GUAG, or asRs-GUAG was subjected to RT extension with a $5^{\prime}$-end-labeled primer complementary to the IRES sequence. cDNA products were analyzed on denaturing $6 \%$ acrylamide gels in parallel with a DNA sequence prepared with the same primer. IRES nucleotides (numbered as in Serrano et al. 2007) are indicated on the left. Arrows denote the position of RT stops specifically detected in the Rz-IRES and Rz-GUAG RNA. Full-length products are show at the top. The relative intensity of RT stops observed in lanes with Rz (measured as the \% of the full-length product) is indicated at the bottom.

RNA. Interestingly, the primer extension stops were located within the region mapped in vitro as the RNase $\mathrm{P}$ recognition motif (Serrano et al. 2007).

The intensity of the RT stops induced by Rz cleavage in the wild-type and the GUAG IRES were determined by densitometry and made relative to the intensity of the fulllength products visualized at the top of the gel (Fig. 7B). The relative intensities of RT stops at nucleotides A167, C192, and C211 were estimated as 4.7, 7.5, and 2.4\%, respectively, in the wild-type RNA. The relative intensity of RT stop observed at C192 in the GUAG IRES represented 10\% of the full-length product. Together, these data are consistent with partial RNA cleavage results obtained by RT-qPCR.

\section{DISCUSSION}

The activity of picornavirus IRES elements is entirely dependent on their structural organization (Martinez-Salas
2008; Belsham 2009). We have previously shown that a structural element encompassing the apical region of domain 3 within the FMDV IRES is recognized in vitro as substrate by the RNA subunit of the cyanobacterial RNase P (Serrano et al. 2007). Importantly, the ribozyme recognition motif is internally located within the FMDV IRES element, by contrast to the HCV and pestivirus IRES (Lyons and Robertson 2003; Sabariegos et al. 2004). Mapping of the cleavage site pointed toward residues located in a bulge within the GNRA stem-loop that may resemble the smallest substrate described for RNase P (Brannvall et al. 2007). The GNRA motif of FMDV as well as that of encephalomiocarditis virus (EMCV) IRES adopts a tetraloop conformation at the tip of a stable stemloop (Fernandez-Miragall and MartinezSalas 2003; Phelan et al. 2004) and mediates the local RNA structure (Fernandez-Miragall et al. 2006). Thus, the Synechocystis PCC6803 rnpB recognition motif within the IRES is located inside a structural element involved in tertiary interactions.

Domain 3 is an integral part of the IRES element, as shown by the experimental demonstration that nucleotide substitutions within this region abrogated IRES activity in both FMDV (Lopez de Quinto and Martinez-Salas 1997) and EMCV (Robertson et al. 1999) RNAs. Moreover, the IRES domains endowed with the capacity to interact with initiation factors eIF4G, eIF4B, and eIF3 (Pilipenko et al. 2000; Lopez de Quinto and Martinez-Salas 2000; Lopez de Quinto et al. 2001) do not exhibit IRES activity (Fernandez-Miragall et al. 2009). Therefore, each domain within the IRES appears to perform a unique specific function during internal initiation although, alone, none of them display IRES activity. Accordingly, transcripts encompassing the full IRES element or at least an entire structural domain can rescue, although partially, the activity of defective elements carrying a mutation in the same domain (Roberts and Belsham 1997; Serrano et al. 2009). This property also applies to domain 3 and, therefore, defines a specific function for this region, different from those performed by domains 2, 4, and 5 .

In this study we have shown the capacity of the cyanobacterial RNase P ribozyme to inhibit IRES activity in tissue culture cells. Inhibition of the FMDV IRES activity 
was dependent on the expression of the active ribozyme RNA subunit; conversely, a double substitution mutant disrupting formation of helix P4 was unable to cleave RNA and, concomitantly, did not inhibit IRES activity. Moreover, expression of an RNA corresponding to the antisense orientation of $r n p B$ did not inhibit IRES activity either. Together, these results demonstrate that stable RNA structural elements located upstream of the IRES element do not interfere with internal initiation activity, fully consistent with the internal location of the IRES element in the $5^{\prime}$ untranslated region of picornavirus viral RNAs. This feature is particularly notable in the aphthovirus RNA where the IRES element is preceded by various highly stable RNA structural motifs involved in essential steps of the virus replication cycle (Fernandez-Miragall et al. 2009).

A strong inhibition of IRES activity was readily detected in constructs expressing the active RNase P, despite only a fraction of the RNA having been cleaved according to the RT-qPCR data, which also was supported by primer extension mapping. These differences may be due to the measurement of distinct parameters: reporter activity or reporter protein synthesis in the case of IRES inhibition and RNA integrity in the case of RT-qPCR. Additionally, there is a possibility that a fraction of the Rz-IRES chimera adopts a trapped RNA structure that is neither cleaved nor active for IRES-dependent translation.

RNA hydrolysis in Rz-IRES construct was detected early upon transfection, suggesting that this event presumably occurs cotranscriptionally and, according to the RNA amounts measured by RT-qPCR with specific primers detecting the nearest downstream IRES region, no apparent RNA degradation was occurring at these early times. The lack of massive RNA degradation led us to propose that the hydrolyzed RNA likely remains associated with IREStransacting factors within cytoplasmic ribonucleoprotein complexes (Martinez-Salas et al. 2008; Fitzgerald and Semler 2009), preserving RNA from unspecific nuclease attack.

We have shown in this study that highly defective IRES mutants with a reorganized RNA structure that are substrates of the ribozyme, as evidenced by both RT-qPCR and primer extension analysis, exhibited a moderate increased translation of the reporter in response to the expression of the RNase P subunit. It is worth noting that cleavage of the defective IRES element yields an uncapped RNA that retains four upstream AUG triplets between the new 5' end and the correct AUG start codon. Subsequent analysis of the translation initiation manner indicated that the increase was due to a switch from IRES-dependent to $5^{\prime}$ end-dependent initiation of the RNase P target RNAs. The basal level of activity of the defective IRES mutants (about $1 \%$ of the wild type) is $5^{\prime}$ independent, and thus, resistant to the action of the Lb protease (Medina et al. 1993; Lopez de Quinto et al. 2002). This feature allowed us to detect a switch in the manner of protein synthesis initiation, which caused an increase of reporter translation. The in- crease, however, did not reach the levels expected for capdependent initiation, and therefore it ruled out the possibility of being initiated from different transcriptional units. It is noteworthy that the switch in translation initiation manner is also in favor of a specific RNA cleavage of the IRES element.

Our data suggest that RNA hydrolysis may interfere with IRES activity through a local RNA structure modification. Given the results obtained with the different IRES elements, it is tempting to suggest that the structural motif recognized by the RNase P must be accessible to the solvent in the cell cytoplasm. In agreement with this idea, in vivo foot-printing studies revealed the accessibility of various residues within the apical region of domain 3 to dimethyl sulfate (Fernandez-Miragall and Martinez-Salas 2007). Moreover, RNA-affinity purification of IRES-bound proteins conducted with soluble cell extracts revealed lower number of factors bound to a transcript encompassing domain 3 than those interacting with the distal domain 5, despite consisting of shorter length (48 versus 210 nucleotides, respectively) (Pacheco et al. 2008). Thus, domain 3 may be crucial for internal initiation because of its RNA structural organization rather than by its interaction with IREStransacting factors. Taken together, these data highlight the functional relevance of the structural motif recognized by RNase P. While it is not known if this motif is physically folding as a tRNA-like motif, there is no doubt of its implication in IRES activity since its mutation by single nucleotide substitution kills the IRES function (FernandezMiragall and Martinez-Salas 2003).

The Picornaviridae family includes a group of pathogens that are the causative agents of important diseases worldwide. Translation of the viral genome, which consists of a positive-sense RNA and is governed by the IRES element, is the first intracellular step of the picornavirus infection cycle. Therefore, the IRES region constitutes a target for antiviral drugs aimed at blocking the virus life cycle (Gutierrez et al. 1994; Vagnozzi et al. 2007). The strong inhibition of the IRES element by expression of the RNase $\mathrm{P}$ in the cytoplasm of transfected cells shown in this study offers a promising antiviral tool to combat picornavirus infections. Interestingly, inhibition of viral pathogenesis by engineered RNase P ribozyme has been shown before (Liu and Altman 1995; Bai et al. 2008), suggesting that this methodology may be extended to other infectious agents. Nucleic acid-based interference provides promising therapeutic agents against many diseases caused by RNA viruses (de los Santos et al. 2005; Lim et al. 2008; Roy et al. 2008; Romero-Lopez et al. 2009). However, these tools show limitations regarding targeting efficiency, sequence specificity, as well as emergence of virus escape variants (Puerta-Fernandez et al. 2003; Gitlin et al. 2005; Schubert et al. 2005; Thompson and Patel 2009). Further studies should examine the activity of RNase P ribozymes for gene targeting applications, including therapy of picornavirus infection. 


\section{MATERIALS AND METHODS}

\section{Constructs}

The plasmid pRz-IRES bearing the FMDV IRES downstream from the Synechocystis sp. PCC6803 ribozyme was constructed by inserting the PCR product encompassing the $r n p B$ gene (RNase $\mathrm{P}$ ribozyme subunit), flanked by PstI restriction sites, replacing CAT in plasmid pBIC (Martinez-Salas et al. 1993). For this, primers Rzas and Rzs (Table 1) and plasmid pT76803 were used in a PCR reaction to obtain the cDNA encoding Synechocystis sp. PCC6803 rnpB gene (Vioque 1992). Similarly, primers Rzs and RzP4as (Table 1) and template Rz-IRES were used to generate a double substitution of bases $\mathrm{GG}_{414-415}$ to $\mathrm{CC}$ in the RNase $\mathrm{P}$ ribozyme (numbers as in Vioque 1992) that abrogate formation of the conserved P4 helix (Pace and Brown 1995).

The defective IRES elements GUAG and CGCCC carrying the indicated nucleotide substitutions in the GNRA and RAAA motifs, respectively (Lopez de Quinto and Martinez-Salas 1997), were constructed following similar procedures. The sequence of constructs expressing the ribozyme (Rz-IRES), the mutant RzP4IRES, or the antisense orientation (asRz-IRES) was determined by automatic sequencing.

\section{Transfection}

BHK-21 cells grown in 24-well dishes were transfected with the pBIC IRES construct $\left(0.33 \mu \mathrm{g} / 10^{5}\right.$ cells $)$ that expresses a bicistronic RNA of the form CAT-IRES-luciferase, or with constructs RzIRES, RzP4-IRES, or asRz-IRES (Fig. 1A) using the T7 RNA polymerase expression system and lipid cationic vehicles as described (Martinez-Salas et al. 1993). In all cases, assays were performed in triplicate wells and in at least three independent assays. Mock-transfected cells were used as negative controls. Cell extracts were prepared $4,8,12$, and $24 \mathrm{hpt}$ (after DNA addition) using $100 \mu \mathrm{L}$ of lysis buffer (50 mM Tris- $\mathrm{HCl}$ at $\mathrm{pH} 7.8,120 \mathrm{mM}$ $\mathrm{NaCl}, 0.5 \% \mathrm{NP} 40$ ). For cells transfected with constructs Rz-IRES, RzP4-IRES, or asRz-IRES, luciferase activity was measured at the indicated times, corrected by the amount of total protein in the cell lysate, determined by the Bradford assay. For Western blot assays, equal amounts of total protein were loaded in 10\% SDSpolyacrylamide gels and then transferred to polyvinylidene fluoride membranes (Bio-Rad) using a semidry electrotransfer device. Identification of the luciferase protein was performed with a rabbit

TABLE 1. Oligonucleotide sequences

\begin{tabular}{llc}
\hline ODN & \multicolumn{1}{c}{ Sequence $\left(5^{\prime}-3^{\prime}\right)$} & Orientation \\
\hline pBICas & GGCCTTTCTTTATGTTTTTGGCG & Antisense \\
Rzs & GGGCTGCAGGAGAGTTAGGGAGG & Sense \\
Rzas & CCCCTGCAGAAAAAAAGAGAGTTAGTC & Antisense \\
RzP4as & GGCCTGCAGAAAAAAAGAGAGTTAGTCGTAAGAAGGG & Antisense \\
T7RTs & GACCGGAAGCTCGACTCTAGAGGATC & Sense \\
T7RTas & TTCGAGGCCACACGCGTCACCTTAATATG & Antisense \\
D3RTs & GGGGTAACACTGAATTCTGTGTTTGGCTCCACG & Sense \\
D3RTas & GCCTGTCACCAGTGTGTGGGTACCAG & Antisense \\
D4-5s & CACACTGAATTCAGGCTAAGGATGCC & Sense \\
Hookas & GGAATGGGATCCTCGAGCTCAGGGTC & Antisense \\
\hline
\end{tabular}

polyclonal antibody (Cortex) at 1:2000 dilution followed by the secondary horseradish peroxidase-conjugated antibody and ECL detection (Amersham). The RNA content of the different IRES transcripts in these samples was determined by RT-qPCR, as described below, in parallel with the IRES-transfected samples.

Plasmid pLb $\left(0.033 \mu \mathrm{g} / 10^{5}\right.$ cells $)$, encoding the FMDV Lb protease, was cotransfected with the different versions of plasmids pBIC (IRES), Rz-IRES, RzP4-IRES, or asRz-IRES $\left(0.2 \mu \mathrm{g} / 10^{5}\right.$ cells) in assays designed to shut down cap-dependent translation (Lopez de Quinto et al. 2002). In transfection assays without pLb, the empty vector pGEM3 was added to provide equal amounts of DNA in the transfection. Cell extracts were prepared 4 and $8 \mathrm{hpt}$. To follow the transfection efficiency, relative IRES activity was quantified as the expression of luciferase normalized to CAT activity in extracts from cells transfected with the IRES constructs (wild type, GUAG, or CGCCC).

\section{RT-qPCR}

The relative amounts of reporter transcript present in total RNA samples prepared from cells $4 \mathrm{hpt}$ were measured by real-time RTPCR. Total RNA from cytoplasmic lysates was isolated using the Tripure reagent (Roche), and RT-PCR was carried out with the SuperScript III RT (Invitrogen) using $50 \mathrm{ng}$ of RNA as template and the reporter-specific primer pBICas (Table 1) as described (Fernandez-Miragall and Martinez-Salas 2007). A control assay in which the RT was omitted was run in parallel to discard the possibility of having plasmid DNA contamination in the total RNA sample isolated from transfected cells. Then, a 1/20 dilution of the RT reaction was used for qPCR using appropriate pairs of specific primers and the LightCycler system with the FastStart DNA Master Green I (Roche) as described (Pacheco et al. 2009). Duplicated samples were analyzed in parallel in three independent assays with two different pairs of FMDV IRES specific primers $(5 \mu \mathrm{M}), \mathrm{D} 3 \mathrm{RT}$ as and D3RTs or D4-5s and Hookas, respectively (Table 1). The amount of RNA detected in this reaction was normalized by a parallel PCR reaction performed with oligonucleotides T7RTs and T7RTas (Table 1), specific for the beginning of the transcript expressed in transfected cells (see Fig. 1B).

\section{Primer extension analysis}

Total cytoplasmic RNA was extracted from transfected cells as described (Fernandez-Miragall and Martinez-Salas 2007), and its concentration was determined by optical density. The antisense primer (5'-CCCG GGTGTGGGTACC-3'), complementary to FMDV IRES, was labeled at the $5^{\prime}$-end using $\left[\gamma-{ }^{32} \mathrm{P}\right]$ ATP and T4 polynucleotide kinase (Boehringer Manheim). Then, total RNA (about $30 \mu \mathrm{g}$ ) was denatured $3 \mathrm{~min}$ at $95^{\circ} \mathrm{C}$ and cooled on ice. Annealing and extension of the labeled primer was carried out in $15 \mu \mathrm{L}$ of RT buffer $(20 \mathrm{mM}$ Tris- $\mathrm{HCl}$ at $\mathrm{pH}$ 7.5, $15 \mathrm{mM} \mathrm{MgCl}_{2}, 100 \mathrm{mM} \mathrm{NaCl}$, $0.1 \mathrm{mM}$ EDTA, $1 \mathrm{mM}$ DTT, $0.01 \%$ [v/v] NP-40, $50 \%[\mathrm{v} / \mathrm{v}]$ glycerol) in the presence of $100 \mathrm{U}$ of Superscript III RT (Invitrogen) and $1 \mathrm{mM}$ each dNTP during $1 \mathrm{~h}$ at $45^{\circ} \mathrm{C}$. The RNA template was then hydrolyzed and RT 
extension products were fractionated in denaturing 6\% acrylamide $7 \mathrm{M}$ urea gels as described (Fernandez-Miragall and MartinezSalas 2003). The sequence ladder was prepared with the same 5 '-labeled antisense oligonucleotide used for primer extension using the Thermosequenase cycle sequencing kit (USB) and plasmid pGEM-IRES as template. The intensity of RT stops was measured in a Bio-Rad GS800 densitometer using the Quantity One program and made relative to the intensity of the respective full-length product detected at the top of the gel in each lane.

\section{SUPPLEMENTAL MATERIAL}

Supplemental material can be found at http://www.rnajournal.org.

\section{ACKNOWLEDGMENTS}

We are grateful to J. Ramajo for technical assistance, and D. Piñeiro, B. Desvoyes, C. Gutierrez, and A. Vioque for helpful suggestions. This work was supported by grant BFU-2008-2159 from MCINN and by an Institutional grant from Fundación Ramón Areces.

Received October 23, 2009; accepted January 4, 2010.

\section{REFERENCES}

Alifano P, Rivellini F, Piscitelli C, Arraiano CM, Bruni CB, Carlomagno MS. 1994. Ribonuclease E provides substrates for ribonuclease P-dependent processing of a polycistronic mRNA. Genes \& Dev 8: 3021-3031.

Andreev DE, Fernandez-Miragall O, Ramajo J, Dmitriev SE, Terenin IM, Martinez-Salas E, Shatsky IN. 2007. Differential factor requirement to assemble translation initiation complexes at the alternative start codons of foot-and-mouth disease virus RNA. RNA 13: 1366-1374.

Bai Y, Trang P, Li H, Kim K, Zhou T, Liu F. 2008. Effective inhibition in animals of viral pathogenesis by a ribozyme derived from RNase P catalytic RNA. Proc Natl Acad Sci 105: 10919-10924.

Belsham GJ. 2009. Divergent picornavirus IRES elements. Virus Res 139: 183-192.

Brannvall M, Kikovska E, Wu S, Kirsebom LA. 2007. Evidence for induced fit in bacterial RNase P RNA-mediated cleavage. J Mol Biol 372: 1149-1164.

Coughlin DJ, Pleiss JA, Walker SC, Whitworth GB, Engelke DR. 2008. Genome-wide search for yeast RNase $\mathrm{P}$ substrates reveals role in maturation of intron-encoded box C/D small nucleolar RNAs. Proc Natl Acad Sci 105: 12218-12223.

de los Santos T, Wu Q, de Avila Botton S, Grubman MJ. 2005. Short hairpin RNA targeted to the highly conserved $2 \mathrm{~B}$ nonstructural protein coding region inhibits replication of multiple serotypes of foot-and-mouth disease virus. Virology 335: 222-231.

Devaney MA, Vakharia VN, Lloyd RE, Ehrenfeld E, Grubman MJ. 1988. Leader protein of foot-and-mouth disease virus is required for cleavage of the p220 component of the cap-binding protein complex. J Virol 62: 4407-4409.

Ellis JC, Brown JW. 2009. The RNase P family. RNA Biol 6: 362-369.

Evans D, Marquez SM, Pace NR. 2006. RNase P: Interface of the RNA and protein worlds. Trends Biochem Sci 31: 333-341.

Fernandez-Miragall O, Martinez-Salas E. 2003. Structural organization of a viral IRES depends on the integrity of the GNRA motif. RNA 9: 1333-1344.

Fernandez-Miragall O, Martinez-Salas E. 2007. In vivo footprint of a picornavirus internal ribosome entry site reveals differences in accessibility to specific RNA structural elements. J Gen Virol 88: 3053-3062.

Fernandez-Miragall O, Ramos R, Ramajo J, Martinez-Salas E. 2006. Evidence of reciprocal tertiary interactions between conserved motifs involved in organizing RNA structure essential for internal initiation of translation. RNA 12: 223-234.

Fernandez-Miragall O, Lopez de Quinto S, Martinez-Salas E. 2009. Relevance of RNA structure for the activity of picornavirus IRES elements. Virus Res 139: 172-182.

Fitzgerald KD, Semler BL. 2009. Bridging IRES elements in mRNAs to the eukaryotic translation apparatus. Biochim Biophys Acta 1789: $518-528$.

Gitlin L, Stone JK, Andino R. 2005. Poliovirus escape from RNA interference: Short interfering RNA-target recognition and implications for therapeutic approaches. J Virol 79: 1027-1035.

Gutierrez A, Martinez-Salas E, Pintado B, Sobrino F. 1994. Specific inhibition of aphthovirus infection by RNAs transcribed from both the $5^{\prime}$ and the $3^{\prime}$ noncoding regions. J Virol 68: 7426-7432.

Kirsebom LA, Trobro S. 2009. RNase P RNA-mediated cleavage. IUBMB Life 61: 189-200.

Ko JH, Altman S. 2007. OLE RNA, an RNA motif that is highly conserved in several extremophilic bacteria, is a substrate for and can be regulated by RNase P RNA. Proc Natl Acad Sci 104: 78157820.

Li Y, Altman S. 2003. A specific endoribonuclease, RNase P, affects gene expression of polycistronic operon mRNAs. Proc Natl Acad Sci 100: 13213-13218.

Lim T, Yuan J, Zhang HM, Sall A, Liu Z, Su Y, Yang D. 2008. Antisense DNA and RNA agents against picornaviruses. Front Biosci 13: 4707-4725.

Liu F, Altman S. 1995. Inhibition of viral gene expression by the catalytic RNA subunit of RNase P from Escherichia coli. Genes \& Dev 9: 471-480.

Lopez de Quinto S, Martinez-Salas E. 1997. Conserved structural motifs located in distal loops of aphthovirus internal ribosome entry site domain 3 are required for internal initiation of translation. J Virol 71: 4171-4175.

Lopez de Quinto S, Martinez-Salas E. 2000. Interaction of the eIF4G initiation factor with the aphthovirus IRES is essential for internal translation initiation in vivo. RNA 6: 1380-1392.

Lopez de Quinto S, Lafuente E, Martinez-Salas E. 2001. IRES interaction with translation initiation factors: Functional characterization of novel RNA contacts with eIF3, eIF4B, and eIF4GII. RNA 7: 1213-1226.

Lopez de Quinto S, Saiz M, de la Morena D, Sobrino F, MartinezSalas E. 2002. IRES-driven translation is stimulated separately by the FMDV 3'-NCR and poly(A) sequences. Nucleic Acids Res 30: 4398-4405.

Lyons AJ, Robertson HD. 2003. Detection of tRNA-like structure through RNase P cleavage of viral internal ribosome entry site RNAs near the AUG start triplet. J Biol Chem 278: 2684426850.

Martinez-Salas E. 2008. The impact of RNA structure on picornavirus IRES activity. Trends Microbiol 16: 230-237.

Martinez-Salas E, Saiz JC, Davila M, Belsham GJ, Domingo E. 1993. A single nucleotide substitution in the internal ribosome entry site of foot-and-mouth disease virus leads to enhanced cap-independent translation in vivo. J Virol 67: 3748-3755.

Martinez-Salas E, Pacheco A, Serrano P, Fernandez N. 2008. New insights into internal ribosome entry site elements relevant for viral gene expression. J Gen Virol 89: 611-626.

Medina M, Domingo E, Brangwyn JK, Belsham GJ. 1993. The two species of the foot-and-mouth disease virus leader protein, expressed individually, exhibit the same activities. Virology 194: 355-359.

Monie TP, Perrin AJ, Birtley JR, Sweeney TR, Karakasiliotis I, Chaudhry Y, Roberts LO, Matthews S, Goodfellow IG, Curry S. 2007. Structural insights into the transcriptional and translational roles of Ebp1. EMBO J 26: 3936-3944. 
Nadal A, Martell M, Lytle JR, Lyons AJ, Robertson HD, Cabot B, Esteban JI, Esteban R, Guardia J, Gomez J. 2002. Specific cleavage of hepatitis C virus RNA genome by human RNase P. J Biol Chem 277: 30606-30613.

Pace NR, Brown JW. 1995. Evolutionary perspective on the structure and function of ribonuclease P, a ribozyme. J Bacteriol 177: 1919-1928.

Pacheco A, Reigadas S, Martinez-Salas E. 2008. Riboproteomic analysis of polypeptides interacting with the internal ribosomeentry site element of foot-and-mouth disease viral RNA. Proteomics 8: 4782-4790.

Pacheco A, Lopez de Quinto S, Ramajo J, Fernandez N, MartinezSalas E. 2009. A novel role for Gemin5 in mRNA translation. Nucleic Acids Res 37: 582-590.

Pascual A, Vioque A. 1999. Substrate binding and catalysis by ribonuclease P from cyanobacteria and Escherichia coli are affected differently by the $3^{\prime}$ terminal CCA in tRNA precursors. Proc Natl Acad Sci 96: 6672-6677.

Phelan M, Banks RJ, Conn G, Ramesh V. 2004. NMR studies of the structure and $\mathrm{Mg}^{2+}$ binding properties of a conserved RNA motif of EMCV picornavirus IRES element. Nucleic Acids Res 32: 47154724 .

Pilipenko EV, Pestova TV, Kolupaeva VG, Khitrina EV, Poperechnaya AN, Agol VI, Hellen CU. 2000. A cell cycledependent protein serves as a template-specific translation initiation factor. Genes \& Dev 14: 2028-2045.

Piron M, Beguiristain N, Nadal A, Martinez-Salas E, Gomez J. 2005. Characterizing the function and structural organization of the $5^{\prime}$ tRNA-like motif within the hepatitis $C$ virus quasispecies. Nucleic Acids Res 33: 1487-1502.

Puerta-Fernandez E, Romero-Lopez C, Barroso-delJesus A, BerzalHerranz A. 2003. Ribozymes: Recent advances in the development of RNA tools. FEMS Microbiol Rev 27: 75-97.

Ramos R, Martinez-Salas E. 1999. Long-range RNA interactions between structural domains of the aphthovirus internal ribosome entry site (IRES). RNA 5: 1374-1383.

Roberts LO, Belsham GJ. 1997. Complementation of defective picornavirus internal ribosome entry site (IRES) elements by the coexpression of fragments of the IRES. Virology 227: 53-62.

Robertson HD, Altman S, Smith JD. 1972. Purification and properties of a specific Escherichia coli ribonuclease which cleaves a tyrosine transfer ribonucleic acid presursor. J Biol Chem 247: 5243-5251.

Robertson ME, Seamons RA, Belsham GJ. 1999. A selection system for functional internal ribosome entry site (IRES) elements: Analysis of the requirement for a conserved GNRA tetraloop in the encephalomyocarditis virus IRES. RNA 5: 1167-1179.

Rodriguez Pulido M, Serrano P, Saiz M, Martinez-Salas E. 2007. Footand-mouth disease virus infection induces proteolytic cleavage of PTB, eIF3a,b, and PABP RNA-binding proteins. Virology 364: 466-474.

Romero-Lopez C, Diaz-Gonzalez R, Barroso-delJesus A, BerzalHerranz A. 2009. Inhibition of hepatitis $C$ virus replication and internal ribosome entry site-dependent translation by an RNA molecule. J Gen Virol 90: 1659-1669.

Roy S, Gupta N, Subramanian N, Mondal T, Banerjea AC, Das S. 2008. Sequence-specific cleavage of hepatitis C virus RNA by DNAzymes: Inhibition of viral RNA translation and replication. $J$ Gen Virol 89: 1579-1586.

Sabariegos R, Nadal A, Beguiristain N, Piron M, Gomez J. 2004. Catalytic RNase P RNA from Synechocystis sp. cleaves the hepatitis C virus RNA near the AUG start codon. FEBS Lett 577: 517522 .

Schubert S, Grunweller A, Erdmann VA, Kurreck J. 2005. Local RNA target structure influences siRNA efficacy: Systematic analysis of intentionally designed binding regions. J Mol Biol 348: 883-893.

Serrano P, Gomez J, Martinez-Salas E. 2007. Characterization of a cyanobacterial RNase $\mathrm{P}$ ribozyme recognition motif in the IRES of foot-and-mouth disease virus reveals a unique structural element. RNA 13: 849-859.

Serrano P, Ramajo J, Martinez-Salas E. 2009. Rescue of internal initiation of translation by RNA complementation provides evidence for a distribution of functions between individual IRES domains. Virology 388: 221-229.

Song Y, Tzima E, Ochs K, Bassili G, Trusheim H, Linder M, Preissner KT, Niepmann M. 2005. Evidence for an RNA chaperone function of polypyrimidine tract-binding protein in picornavirus translation. RNA 11: 1809-1824.

Thompson AJ, Patel K. 2009. Antisense inhibitors, ribozymes, and siRNAs. Clin Liver Dis 13: 375-390.

Vagnozzi A, Stein DA, Iversen PL, Rieder E. 2007. Inhibition of footand-mouth disease virus infections in cell cultures with antisense morpholino oligomers. J Virol 81: 11669-11680.

Vioque A. 1992. Analysis of the gene encoding the RNA subunit of ribonuclease P from cyanobacteria. Nucleic Acids Res 20: 63316337.

Yang L, Altman S. 2007. A noncoding RNA in Saccharomyces cerevisiae is an RNase P substrate. RNA 13: 682-690. 

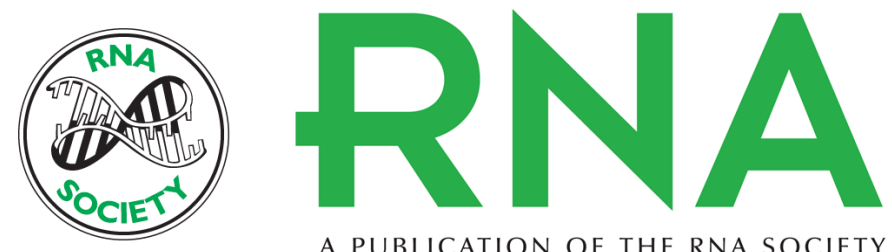

A PUBLICATION OF THE RNA SOCIETY

\section{Tailoring the switch from IRES-dependent to 5'-end-dependent translation with the RNase P ribozyme}

Noemi Fernández and Encarnación Martínez-Salas

RNA 2010 16: 852-862 originally published online March 1, 2010

Access the most recent version at doi:10.1261/rna.1973710

\section{Supplemental http://rnajournal.cshlp.org/content/suppl/2010/02/09/rna.1973710.DC1 \\ Material}

References This article cites 57 articles, 28 of which can be accessed free at:

http://rnajournal.cshlp.org/content/16/4/852.full.html\#ref-list-1

\section{License}

Email Alerting Receive free email alerts when new articles cite this article - sign up in the box at the Service top right corner of the article or click here. 